\title{
Duration of looking and number of brief looks as dependent variables'
}

DAVID PREMACK, UNIVERSITY OF CALIFORNIA, SANTA BARBARA GEORGE COLLIER, RUTGERS UNIVERSITY

The duration for which human Ss exposed themselves to stimuli before responding on simultaneous discrimination problems varied with stimulus difficulty, discrimination reversal and reinforcement schedule.

In many situations in which $S^{\prime} s$ task is to make a discrimination, no knowledge of the course of learning can be obtained since only success or failure can be recorded. The hypothesis that some intensive property of "looking" can provide an index of the time-course of the formation of a discrimination is examined in the present experiment.

Once $S$ looks on every trial, the standard digital measure of the observing response (e.g., Skinner, 1953; Wyckoff, 1952) precludes detecting further variation in looking. However, it seems certain that the frequency of looking at a stimulus of short duration or the duration of a look at a stimulus of subject-controlled duration will vary as a function of the parameters of discrimination.

\section{Method}

Human Ss, obtained from introductory psychology, were instructed in the use of a stimulus control button and tested on simultaneous discrimination problems. The $\mathrm{S}$ was seated before a screen on which the stimuli were projected and was provided with three keys. The center key presented the stimuli and the two side keys registered the choice. Lights above the side keys indicated correct choices. Two procedures were used. In the first, operation of the center (stimulus) key presented the stimuli for $0.2 \mathrm{sec}$. , and $\mathrm{S}$ was allowed an unlimited number of these exposures per discrimination trial. Frequency per trial was recorded. In the second procedure, the stimulus key exposed the stimuli for the duration of the key depression. Durations were recorded. The stimulus material consisted of sets of 10 by 10 grids with varying degrees of randomlyselected fill.

\section{Resulis}

The effect of stimulus difficulty was explored by two problems in both of which the two grids were $50 \%$ filled but with an $85 \%$ overlap of the grids in the "hard" problem and only a $15 \%$ overlap in the "easy" problem. Both problems were presented to the same six Ss, "hard"-"easy" for three Ss, "easy"-"hard" for the other three, and with the second problem given immediately after the first.

Figure 1 shows duration of stimulus exposure as a function of blocks of successive five trials, with separate curves for the two problems. Both the effects of problems and trials were significant $(p<.01)$, though

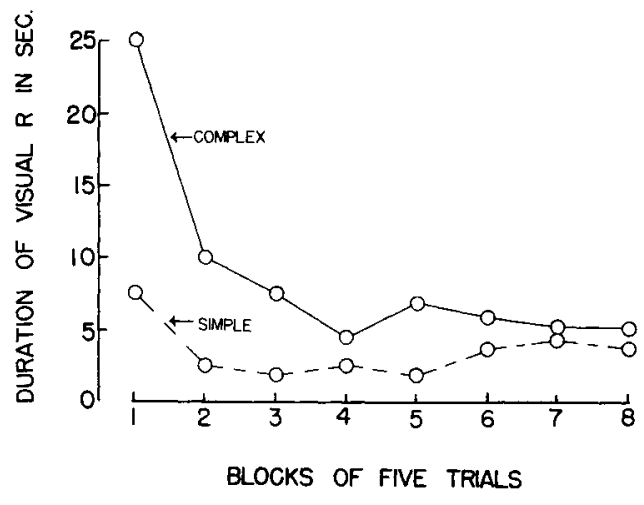

Fig. 1. Effect of stimulus complexity on looking time

the $\mathrm{P}$ by $\mathrm{T}$ interaction did notattain the $5 \%$ level. Initial looking times clearly differed in the expected direction but the more interesting question of asymptotic difference will require further tests.

Both curves in Fig. 1 show a gradual decline in looking time over trials. These data are not wholly representative of individual performance where, more typically, a long early look-not necessarily on the first trialwas followed by a nearly asymptotic decline. Figure 2 shows six representative records for experienced Ss, three for duration procedure, three for number procedure. The problems were of the same grid type. The naive human $S$ often produces a later stimulus exposure equal to or even longer than an earlier one, but such performance is rare in the experienced $S$.

The effect of discrimination reversal was studied with 24 Ss who were given a reversal on trial 21 following 20 standard discrimination trials. As shown in Fig. 3, which plots exposure time and choice responses averaged over all Ss per block of five trials, the reversal occasioned a clearcut increase in both exposure time and discrimination errors. Interestingly, the looking duration produced by the reversal did not equal that found at the beginning of the problem. Both errors and looking time increased together; this relation was examined in the next experiment by manipulating reinforcement schedule.

Three groups of six Ss each were given the same discrimination problem in conjunction with a different reinforcement schedule. The schedules were: one stimulus always correct, the other always incorrect (differential reinforcement); both stimuli always correct (nondifferential reinforcement); both stimuli correct $50 \%$ of the time at random with neither correct more than twice in a row (random reinforcement). 

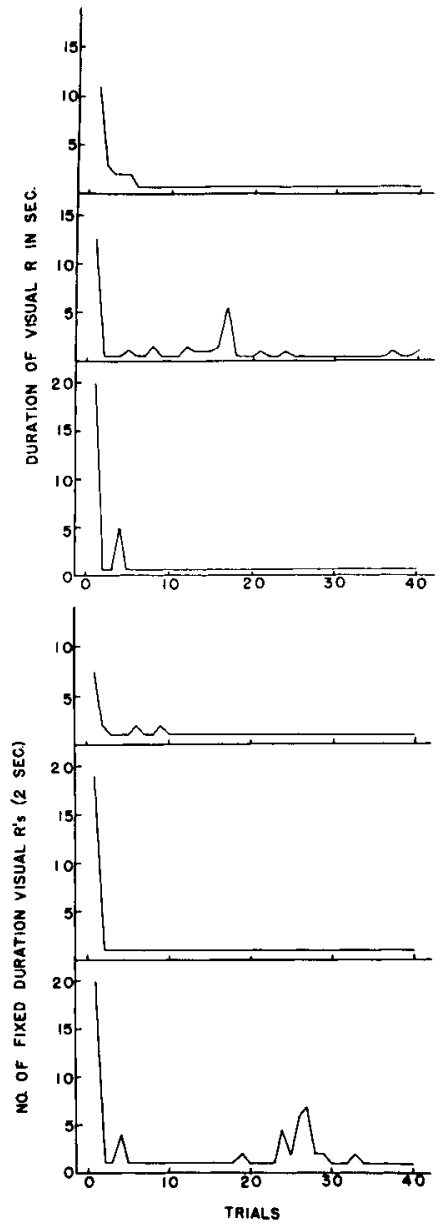

Fig. 2. Representative individual records

Figure 4 shows duration of looking as a function of blocks of five trials with separate curves for the three reinforcement groups. Looking time was least for the group that was nondifferentially reinforced, intermediate for the standard differential condition, and greatest for the random condition. Interestingly, exposure time decreased over trials for the random condition as well as for the other conditions. The main effects of groups and trials were both significant $(p<.01)$, while the $\mathrm{G}$ by $\mathrm{T}$ interaction did not reach the $5 \%$ level. $t$-tests showed all groups except the differential and nondifferential to differ by at least the $5 \%$ level.

\section{Conclusions}

These results, indeed, show that two intensive properties of looking are sensitive to such variables as

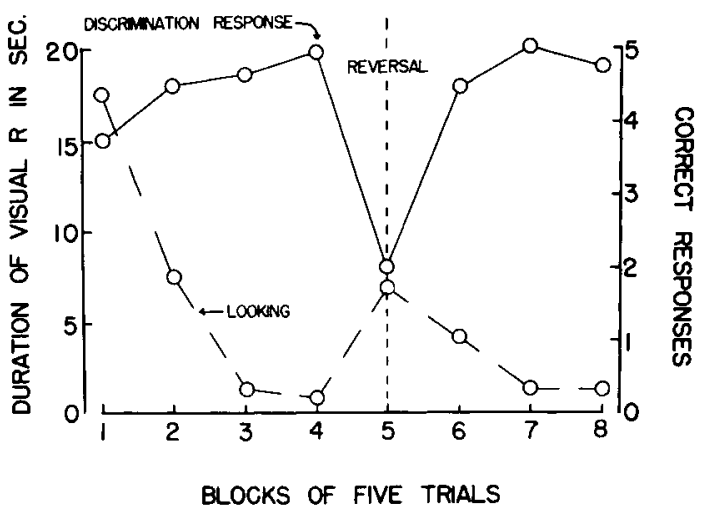

Fig. 3. Effect of discrimination reversal on looking time and instrumental choice

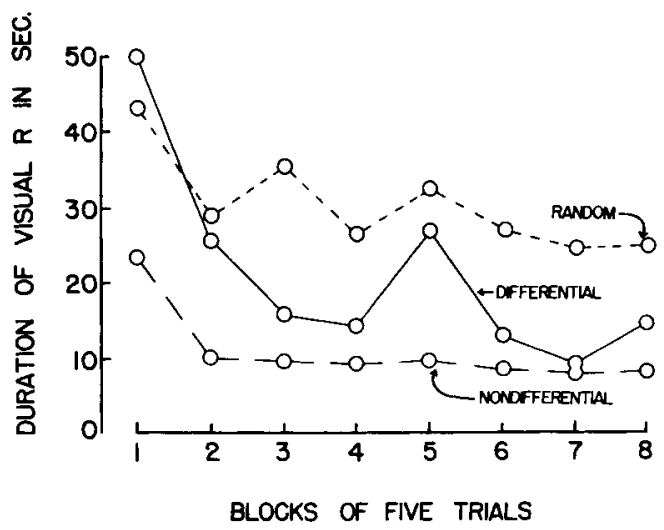

Fig. 4. Effect of reinforcement schedule on looking time

practice, difficulty, and reinforcement, commonly used in discrimination studies. The large difference between initial looking durations and those observed at the time of the reversals in the discrimination reversal problem suggest that perhaps a two stage problem is involved in looking. The first is familiarization with the range of stimuli involved, the second is the solution of the problem, the latter requiring briefer or fewer looks since it requires only a selection from a limited number of possibilities.

\section{References}

Skinner, B. F. Are theories of learning necessary? Psychol. Rev., $1950,57,193-216$.

Wyckoff, L. B. Jr. The role of observing responses in discrimination learning: Part 1. Psychol. Rev., 1952, 59, 431-442.

\section{Noie}

1. Report prepared with the aid of NIH grant MH 11930. 